\title{
A Novel Selection Circuit Based on Rough Comparison Method for Genetic Algorithms Hardware
}

\author{
Tomokazu Hiratsuka Student Member (Kyushu Inst. of Tech., hiratsuka-tomokazu@edu.brain.kyutech.ac.jp) \\ Hakaru Tamukoh Non-member (Tokyo Univ. of Agriculture and Tech., tamukoh@cc.tuat.ac.jp) \\ Keiichi Horio Non-member (Kyushu Inst. of Tech., horio@brain.kyutech.ac.jp) \\ Takeshi Yamakawa Non-member (Kyushu Inst. of Tech., yamakawa@brain.kyutech.ac.jp)
}

Keywords : genetic algorithm, selection scheme, rough comparison method, digital hardware

Genetic algorithm (GA) are search techniques used in computing to find true or approximate solutions to optimization and search problems. GA are a particular class of evolutionary algorithms that use techniques inspired by evolutionary biology such as inheritance, selection, crossover and mutation. GA are categorized as global search heuristics.

Hardware accelerators for GA are required to reduce the execution time. Therefore, many research results for hardware implementation of GA have been reported. In the hardware implementation of GA, a circuit design of roulette wheel selection influences the performance of the GA hardware.

This paper propose a new selection circuit based on Rough Comparison Method (RCM). The RCM selects the larger data included within a certain definite range as selection candidates (SC). Figure 1 illustrates an example of the RCM. In the data $\left(D_{0}\right.$ $\sim D_{5}$ ), the high-order several bits which are defined Range are used for data comparison. The other bits are considered as a rounding error. When the high-order four bits are compared (i.e. Range $=4)$, the values of $D_{0}, D_{4}$ and $D_{5}$ are 112. Thus, $D_{1}, D_{2}$ and $D_{3}$ is ruled out as SC, because the value of $D_{1}, D_{2}$ and $D_{3}$ is 96,80 and 16 , respectively. The larger data in the data group is selected by this procedure. If there are several data in the SC, only one data is selected at random from the SC. If the fitness values of GA are used to RCM, RCM selects one fitness value from larger fitness values at random. i.e. the individuals which have larger fitness are selected in high probability. Therefore RCM's operation is as good as the ordinary roulette wheel selection scheme.

Figure 2 illustrates the proposed roulette selection circuit based on the RCM. This is a parallel processing circuit. Part (A) searches the individuals of SC. First, shift left registers (data) store the fitness values of all the individuals, and flag of all cells are set to '1.' Secondly, when at least one of the MSBs is '1,' signal line becomes ' 0 .' Then, the data of MSB $=0$ is smaller than that of MSB $=1$. Therefore, the flag of the data of MSB $=0$ changes to ' 0 .' i.e. the data are no longer SC. Finally, data is shifted to the left. These operations are repeated Range times. The Range is the parameter which represents accuracy of the selection. In other words, the Range determines the threshold of the fitness. Part (A) takes a lot of SC when the Range is small. Part (B) selects only one individual at random from the SC. Only one cell receives rand $_{\mathrm{j}}=1$, and others ' 0 ' at random by the random number generator. When the rand $_{\mathrm{j}}=1$ and flag $_{\mathrm{j}}=1$ or $C_{\mathrm{in}, \mathrm{j}}=1$ and flag $_{\mathrm{j}}=$ $1, w^{\prime} n_{\mathrm{j}}$ changes to ' 1 ,' otherwise ' 0 .' i.e. $j$-th individual is selected as the individual to be used in the next process of GAs. If the flag $_{j}$ $=0$ and $\operatorname{rand}_{\mathrm{j}}=1$, the chance to be selected is ceded to the next
$\operatorname{cell}\left(C_{\text {out }, \mathrm{j}}=1\right)$.

We designed a GA hardware embedded the proposed new selection circuit based on the RCM. The software simulations results showed that the proposed selection scheme has a performance better than the ordinary roulette wheel selection scheme. The hardware implementation result showed that the proposed circuit has a considerable validity. Moreover, the execution time and circuit size of the proposed circuit can be reduced. Therefore the proposed circuit suits well for the GA hardware.

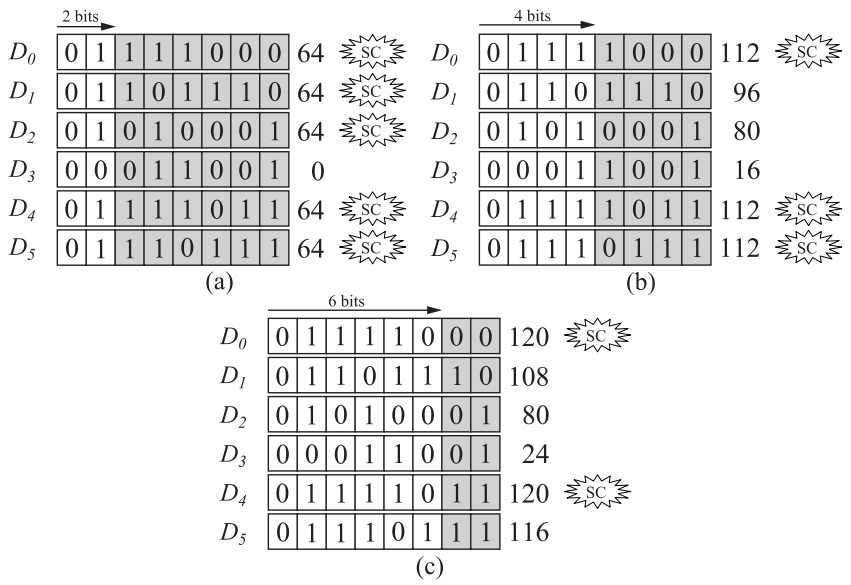

Fig. 1. An architecture of the rough comparison method; (a) Range $=2$, (b) Range $=4$, (c) Range $=6$

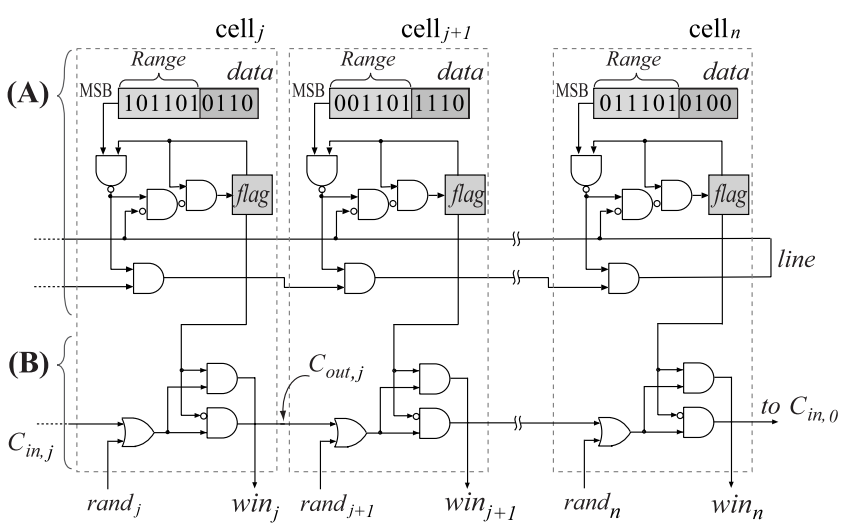

Fig. 2. RCM circuit; part (A) searches the SC, part (B) selects one individual from $\mathrm{SC}$ 


\title{
A Novel Selection Circuit Based on Rough Comparison Method for Genetic Algorithms Hardware
}

\author{
Tomokazu Hiratsuka* Student Member \\ Hakaru Tamukoh** Non-member \\ Keiichi Horio* \\ Takeshi Yamakawa* $\quad$ Non-member
}

\begin{abstract}
Genetic algorithms (GAs) are search algorithms based on the mechanics of natural selection and natural evolution. Due to cost reasons, hardware accelerators for GAs are required to reduce its execution time. In the hardware implementation of GAs, a circuit design of roulette wheel selection influences the performance of the GAs hardware. In this paper, we propose a new selection circuit based on Rough Comparison Method (RCM), and evaluate effects of the proposed circuit in terms of execution time and circuit size. The RCM is a parallel processing circuit. Therefore, the execution time is constant regardless of the increase of number in individuals, and the circuit size of the RCM is reduced than the ordinary parallel roulette selection circuits.
\end{abstract}

Keywords: genetic algorithm, selection scheme, rough comparison method, digital hardware

\section{Introduction}

Genetic algorithm (GA) is a search technique used in computing to find true or approximate solutions to optimization and search problems ${ }^{(1)(2)}$. GA is a particular class of evolutionary algorithm that uses techniques inspired by evolutionary biology such as inheritance, selection, crossover and mutation. GA is categorized as global search heuristic. GA has been applied to many hard optimization problems in computer science, engineering, economics, physics, mathematics and other fields ${ }^{(3)}$. They have been recognized as a robust general purpose optimization technique.

However, application of GA to increasingly complex problems can overwhelm software implementations of GA, causing unacceptable delays in the optimization process. This is true of any non-trivial application of GA if the search space is large. It follows that a hardware implementation of GA would be applicable to problems too complex for software-based GA.

In addition, a personal navigation system for tourism (4) and a high resolution image compression system ${ }^{(5)}$ are proposed as an application of GA to embed in a portable terminal. A speedup, a miniaturization and a low-power consumption of GA are fundamental to embed these applications in the portable terminal.

Therefore, many research results for hardware implementation of GA have been reported ${ }^{(6)-(15)}$. Although these hardware accelerate execution of GA over soft-

\footnotetext{
* Graduate School of Life Science and Systems Engineering, Kyushu Institute of Technology

2-4, Hibikino, Wakamatsu, Kitakyushu, 808-0196

** Institute of Symbiotic and Technology, Tokyo University of

Agriculture and Technology

2-24-16, Naka-cho, Koganei-city, Tokyo, 184-8588
}

ware implementations, the speedup and the miniaturization of the circuits are not enough. Because these hardware were embedded of a roulette wheel selection circuit which is a major bottleneck with respect to the execution time and the circuit size.

In this paper, we proposed a new selection circuit based on a rough comparison method (RCM) to improve the performance of the selection circuit on the speedup of the execution time and the downsizing of the circuit size.

This paper is organized into seven sections. Section 2 provides a brief overview of genetic algorithms and extracts problem of previously reported GA hardware. Section 3 describes the rough comparison method. Section 4 discusses software simulations of GA using a proposed selection algorithm. Section 5 describes the GA hardware embedded proposed selection algorithm. Section 6 discusses a Range value and a optimization problem. Finally, Section 7 contains some concluding remarks.

\section{Genetic Algorithm}

2.1 Basic Architecture GA is an optimization technique based on the process of natural selection and evolution $^{(1)}$. A simple genetic algorithm (SGA) is the basis of $\mathrm{GA}^{(2)}$. The SGA has shown its usefulness for optimization problems requiring the search of large and complex problem spaces from engineering design to combinatorial optimization to control.

The SGA maintains a collection (population) of candidate solutions during the SGA operation. Associated with each candidate is a fitness or measure of its quality. An algorithm of the SGA proceeds by selecting candidates from the current generation to propagate into the next generation. In the process of this propagation the 


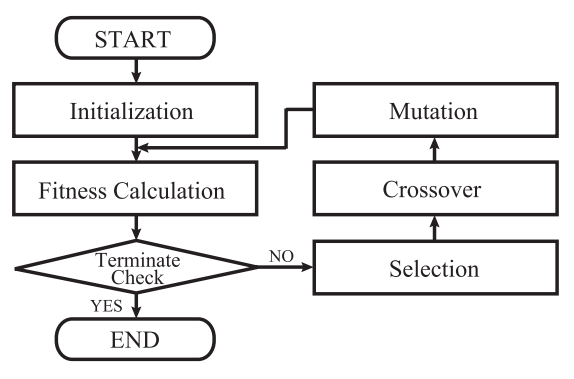

Fig. 1. A flow of SGA

algorithm may simply copy the selected candidates to the new generation or it may combine pairs of candidates through a crossover operation, reminiscent of mating in natural systems. In this case, the newly created the candidate solutions have characteristics taken from both parents. The selection of candidates for copy and crossover is randomized but biased toward candidates with higher fitness, thus, more fit individuals are more likely to be used to produce future generations of solutions. As a means of preventing premature convergence to local minima, an operation known as mutation randomly perturbs solutions to yield new ones not otherwise related to existing solutions.

SGA works as follows;

(1) Randomly generating an initial population $P_{0}$

(2) Computing and saving the fitness $f_{i}$ for each individual $G_{i}$ in the current population $P_{t}$

(3) Defining selection probabilities $p_{i}$ for each individual $G_{i}$ in $P_{t}$ so that $p_{i}$ is proportional to $f_{i}$

(4) Generating $P_{t+1}$ by probabilistically selecting individuals from $P_{t}$ to produce offspring via genetic operators (crossover, mutation)

(5) Repeating step 2 to 4 until satisfying solution is obtained.

These steps are summarized in Fig. 1.

2.2 Hardware Implementation of GA A hardware implementation of GA is effective to obtain drastic performance improvement. The structure of GA computation forms a good basis for hardware. There have already been research carried out on hardware for GA, some of which concerned specific problems such as the pattern matching ${ }^{(6)}$, the scheduling ${ }^{(7)}$, the traveling salesman problem ${ }^{(8)}$ and the image filtering ${ }^{\left({ }^{(9)}\right.}$, and some of which are a general-purpose hardware ${ }^{(10)-(15)}$.

GA hardware which we propose belongs to the group of the general-purpose hardware.

2.3 Problems of Previous Works Much GA hardware which was reported previously employs a roulette wheel selection scheme for individual selection operation.

The roulette wheel selection is a representative stochastic selection model which is based on a fitnessproportionate selection strategy. The principle of the roulette wheel selection is a linear search over a roulette wheel with slots weighted in proportion to the individual's fitness value. At first, a sum of the fitness is calculated in Eq. (1), and then, a target value $S_{t}$ set which is a random proportion of the sum of the fitness values

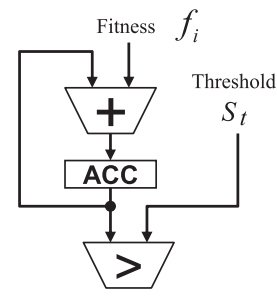

(a)

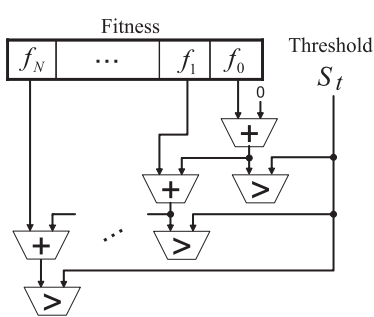

(b)
Fig. 2. Selection circuits of the ordinary roulette wheel selection ${ }^{(8)}$ (main parts): (a) serial processing circuit, (b) parallel processing circuit

in the population in Eq. (2). The population is stepped through until the target value is reached. If the best individual is not selected to reproduce or if they're destroyed by crossover or mutation, they will be lost. To solve this problem, an elitist preserving selection is an addition to many selection methods that forces the GA to retain some number of the best individuals at each generation.

Procedure of the roulette wheel selection is as follows;

(1) Summing up the fitness values of all individuals within the current population.

$$
S_{N}=\sum_{i=1}^{N} f_{i} \ldots \ldots \ldots \ldots \ldots \ldots \ldots \ldots
$$

(2) Generating a random number rand $\in[0,1]$ and calculating the target value $S_{t}$.

$$
S_{t}=\operatorname{rand} \times S_{N} \cdots
$$

(3) Examining the individuals in the order in which they appear in the population, and accumulating the fitness values into $S_{a}$ up to $k$ times where $k$ is number of individuals. The individual under examination is selected when $S_{a}>S_{t}$.

$$
S_{a}=\sum_{i=1}^{k} f_{i} \geq S_{t} \ldots \ldots \ldots \ldots \ldots \ldots
$$

This method is implemented in two kinds of circuits ${ }^{\left({ }^{8}\right)}$; serial processing circuit and parallel processing circuit (Fig. 2). However, these circuits (a) and (b) are major bottleneck with respect to the execution time and the circuit size, respectively.

Hence, several studies have focused on the improvement of selection scheme. Asada et al. ${ }^{(13)}$ suggested "roulette selection with binary search." This selection scheme narrowed the search space of the target individuals in roulette wheel selection by binary search to reduce of execution time. Besides, Yoshida et al. ${ }^{(15)}$ suggested "simplified tournament selection." This selection scheme reduced the execution time by simplification of a tournament selection scheme. This scheme selects two individuals at random, and the better one reproduces to next generation.

We propose a RCM-based selection scheme to improve the performance of the selection circuit on the speedup of execution time and the downsizing of the circuit size. And we will discuss performance comparisons between the RCM and the previous works in Sec. 4 and 5.3. 


\section{RCM-Based Selection}

3.1 RCM The rough comparison method (RCM) selects the larger data included within a certain definite range as selection candidates $(\mathrm{SC})^{(16)}$. Figure 3 illustrates an example of the RCM. In the data $\left(D_{0} \sim D_{5}\right)$, the high-order several bits which are defined as a Range are used for data comparison. The other bits are considered as a rounding error.

As shown in Fig. 3, when the high-order one bit is compared, all data are selected as SC. Because each bit is '0.' When the high-order two bits are compared, the values of every data except for $D_{3}$ are 64 . At this point, as $D_{3}$ is smaller than the other data, $D_{3}$ is ruled out as SC. When the high-order four bits are compared, the values of $D_{0}, D_{4}$ and $D_{5}$ are 112 . Thus, $D_{1}, D_{2}$ and $D_{3}$ is ruled out as SC, because the value of $D_{1}, D_{2}$ and $D_{3}$ is 96, 80 and 16, respectively. If all bits are compared, the maximum data is selected. The larger data in the data group is selected by this procedure. If there are several data in the SC, only one data is selected at random from the SC.

3.2 Selection Scheme Using RCM As mentioned above, RCM selects the larger data at random. If the fitness values of $\mathrm{GA}$ are used to RCM, RCM selects one fitness value from larger fitness values at random. i.e. the individuals which have larger fitness are selected in high probability from all the individuals. Therefore RCM's operation is as good as the ordinary roulette wheel selection scheme. When it is necessary to select several individuals from the population, the $\mathrm{RCM}$ only selects several times from the SC at random. Thus the execution time for selection scheme can be reduced. Moreover, the population of next generation is composed of the individuals which are selected from the $\mathrm{SC}$ at random.

The RCM-based roulette wheel selection drastically reduces the execution time. Because this selection scheme takes only Range times by selecting one individual although the ordinary roulette wheel selection takes up to number of individuals times. In addition, the bit comparison in the RCM is well suited for a digital hardware implementation.

\section{Simulations and Results}

4.1 Verification of Effectiveness In order to verify the effectiveness of the proposed selection scheme based on the RCM in SGA, it is applied to four test problems ${ }^{(17)(18)}$; Eq. (4) is parabola, Eq. (5) is step function, Eq. (6) is Shekel's foxholes ( $a_{i j}$ is parameter) and Eq. (7) is Rastrigin function. The sketches of these functions are shown in Fig. 4.

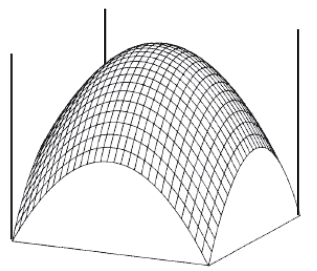

(a)

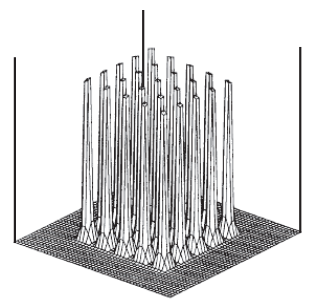

(c)

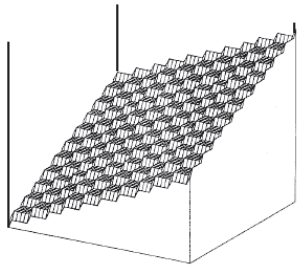

(b)

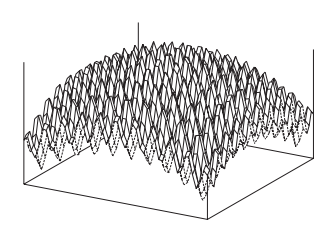

(d)
Fig. 4. Test functions (two-dimensional version) ${ }^{(17)(18)}$; (a) parabola, (b) step function, (c) Shekel's foxholes, (d) Rastrigin function

Table 1. GA parameters in software implementation

\begin{tabular}{|c|c|}
\hline No. of individuals & 64 \\
\hline Precision of individual & $\begin{array}{l}\text { (4) } 30 \text { bits } \\
\text { (5) } 50 \text { bits } \\
\text { (6) } 34 \text { bits } \\
\text { (7) } 100 \text { bits }\end{array}$ \\
\hline Precision of fitness & 16 bits \\
\hline Probability of crossover / mutation & $0.3 / 0.05$ \\
\hline Range (in proposed method) & 10 bits \\
\hline No. of generations & $\begin{array}{c}(4)-(6) 500 \\
(7) 10000\end{array}$ \\
\hline
\end{tabular}




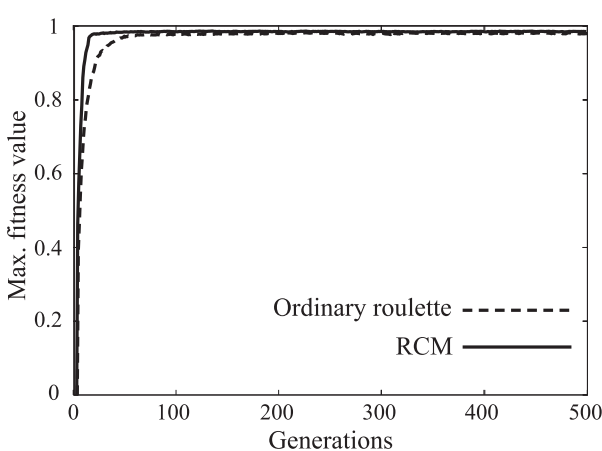

(a)

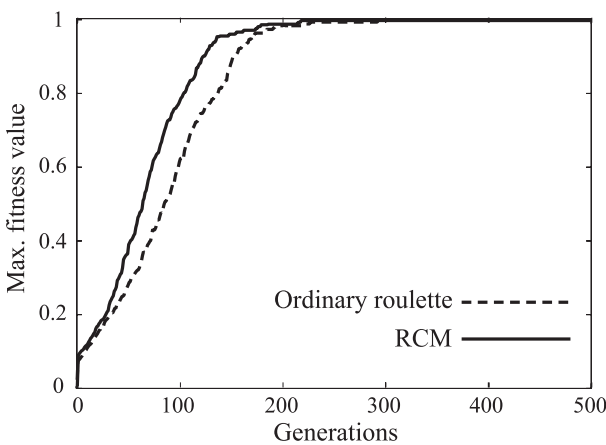

(b)

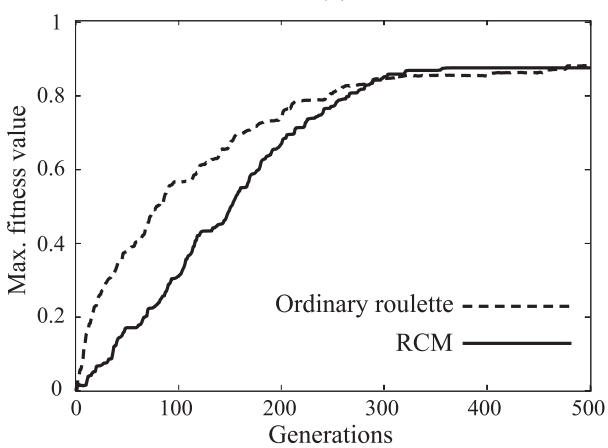

(c)

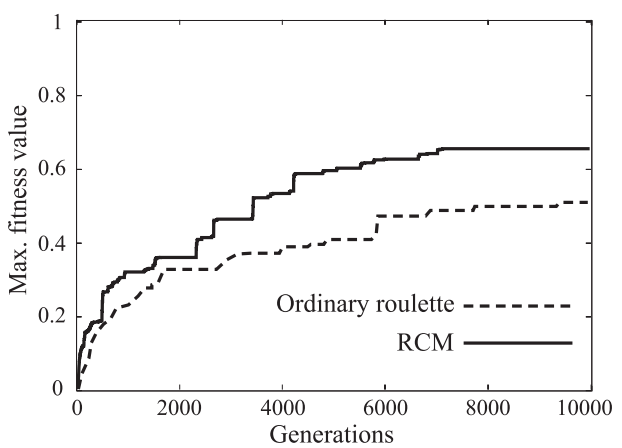

(d)

Fig. 5. Comparison of selection schemes in software implementation; (a) parabola, (b) step function, (c) Shekel's foxholes and (d) Rastrigin function; these are average of 100 trials of each 500 and 10000 generations

$$
\begin{aligned}
& f(x)=-\sum_{i=1}^{3} x_{i}^{2} \quad\left(-5.11 \leq x_{i} \leq 5.12\right) \cdots \cdots \\
& f(x)=-\sum_{i=1}^{5}\left\lfloor x_{i}\right\rfloor \quad\left(-5.11 \leq x_{i} \leq 5.12\right) \cdots \cdots
\end{aligned}
$$

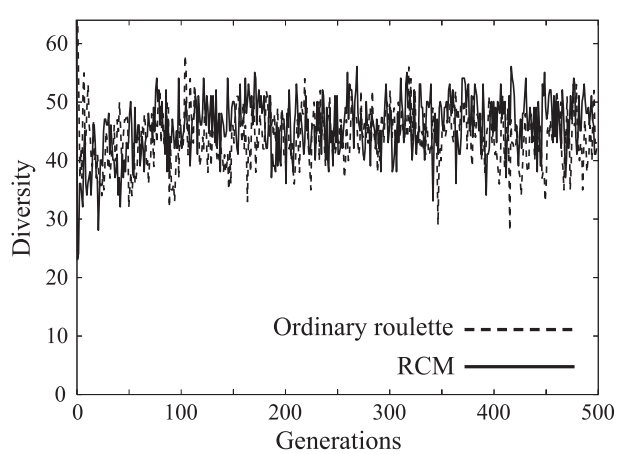

(a)

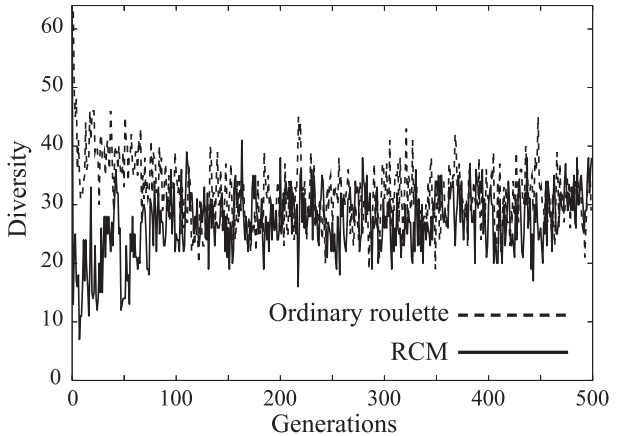

(b)

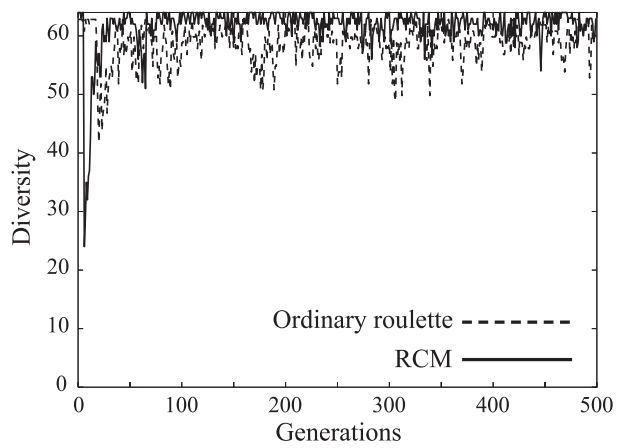

(c)

Fig. 6. Comparison of population diversity; (a) parabola, (b) step function, (c) Shekel's foxholes

$$
\begin{aligned}
f(x)=-\left[\frac{1}{500}+\right. & \left.\sum_{j=1}^{25} \frac{1}{j+\sum_{i=1}^{2}\left(x_{i}-a_{i j}\right)^{6}}\right]^{-1} \\
& \left(-65.535 \leq x_{i} \leq 65.536\right) \cdots \\
f(x)=-[100+ & \left.\sum_{i=1}^{10}\left(x_{i}^{2}-10 \cos \left(2 \pi x_{i}\right)\right)\right] \\
& \left(-5.11 \leq x_{i} \leq 5.12\right) \cdots \cdots
\end{aligned}
$$

The convergence property of the proposed selection was compared with the ordinary roulette wheel selection scheme. We programmed both the proposed selection and the ordinary selection schemes in software implementation. In these simulations, parameters of GA were configured as shown in Table 1.

Figure 5 illustrates the results of simulations. These results are represented as the maximum fitness values of each generation, and are average of 100 trials of each 500 and 10000 generations. As shown in Fig. 5, we confirmed that the convergence property of the proposed selection scheme is better than the ordinary roulette wheel selec- 


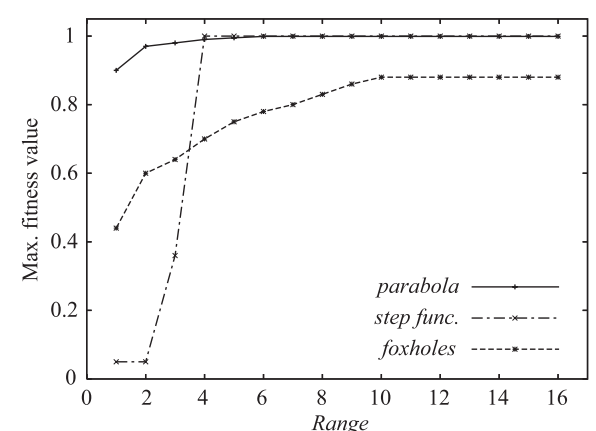

(a)

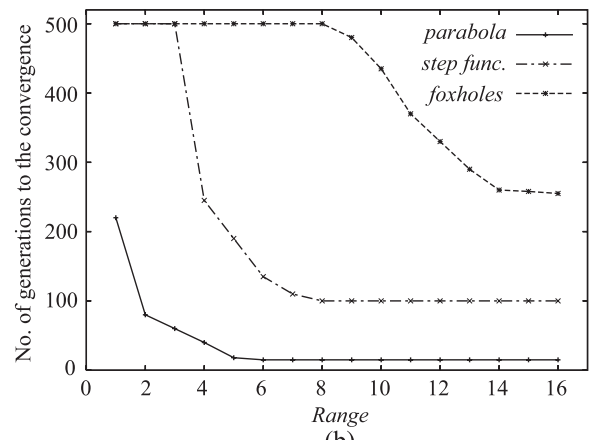

(b)

Fig. 7. The maximum fitness values and the number of generations to the convergence when the Range value was set from 1 to 16; (a) a relationship between the maximum fitness value and the Range, (b) a relationship between the convergence speed (generations) and the Range

tion scheme.

The Rastrigin function and the Shekel's foxholes are hard problem for SGA. In order to resolve these hard problems, new GA architectures such as a parallel GA ${ }^{(2)}$ have been proposed. These GA embedded new architecture for upgrading a searching ability. Yet the ordinary selection scheme such as the roulette wheel selection is used. Thus, the new GA architecture with the RCM-based selection scheme will be able to resolve the hard problem for SGA because the RCM-based selection scheme is better than the ordinary roulette wheel selection scheme.

4.2 Verification of Diversity When only one $\mathrm{SC}$ exists in the population, it is quite likely that a population diversity is reduced because the same SC is kept selecting. Figure 6 shows a transition of the population diversity in one trial of each test problem. In these simulations, parameters of GA were configured as shown in Table 1. As shown in Fig. 6, it is certain that the population diversity is maintained by the RCM-based selection scheme.

Therefore, the proposed selection scheme is a valid method for the selection operation of GA.

4.3 How to Set the Range Figure 7 illustrates the maximum fitness values and the number of generations to the convergence when the Range value was set from 1 to 16 . These results are average of 100 trials, where 500 generations are performed for 1 trial.

As shown in Fig. 7(a), the maximum fitness values of the test functions parabola, step function and

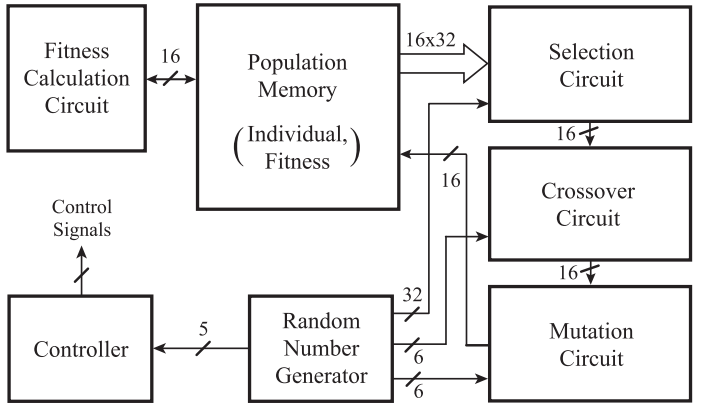

Fig. 8. Block diagram of GA hardware

Table 2. GA parameters in hardware implementation

\begin{tabular}{c|c}
\hline No. of individuals & 32 \\
\hline Precision of individual & 16 bits \\
\hline Precision of fitness & 16 bits \\
\hline Probability of crossover / mutation & $0.2 / 0.01$ \\
\hline Range & 10 bits \\
\hline No. of generations & 10 \\
\hline
\end{tabular}

Shekel's foxholes converges above Range $=6,4$ and 10, respectively. Moreover, as shown in Fig. 7(b), the number of generations to the convergence of the test functions parabola, stepfunction and Shekel's foxholes converges above Range $=5,8$ and 14, respectively.

Accordingly, we adopted empirically the Range $=10$ with careful regard to the results in Fig. 7. If we want to speed up the convergence speed, we can set the large Range value. Alternatively, the circuit requires a long execution time to select individuals.

\section{Digital Hardware Design}

5.1 System Architecture We designed a GA hardware embedded the proposed selection algorithm. Figure 8 illustrates a block diagram of a proposed GA hardware. This hardware implements SGA architecture, and consists of a global controller, a population memory, a random number generator, a fitness calculation circuit, a selection circuit, a crossover circuit and a mutation circuit. Table 2 shows the GA parameters in hardware implementation.

Each part of GA hardware is described in the following section.

5.1.1 Random Number Generator ${ }^{(10)}$ The random number generator supplies pseudo-random bit strings to the selection, crossover and mutation circuit.

The random number generator uses a linear cellular automata $(\mathrm{CA})^{(20)}$ to generate a sequence of pseudorandom bit strings, and is embedded in some previous GA hardware ${ }^{(10)(12)(15)}$. The CA used in the random number generator consists of some alternating cells (bits) which change their states according to rules named "90" and "150":

$$
\begin{aligned}
& \text { Rule } 90: S_{i}^{+}=S_{i-1} \oplus S_{i+1} \ldots \ldots \ldots \ldots \ldots \ldots \\
& \text { Rule } 150: S_{i}^{+}=S_{i-1} \oplus S_{i} \oplus S_{i+1} \ldots \ldots \ldots \ldots
\end{aligned}
$$

where $S_{i}$ is the current state of the $i$-th cell (bits) in the linear cell array (bit string), $S_{i}^{+}$is the next state for $S_{i}$, and $\oplus$ is the exclusive OR operator. Serra et al. ${ }^{(20)}$ showed that a 16 -cell CA whose cells are updated 


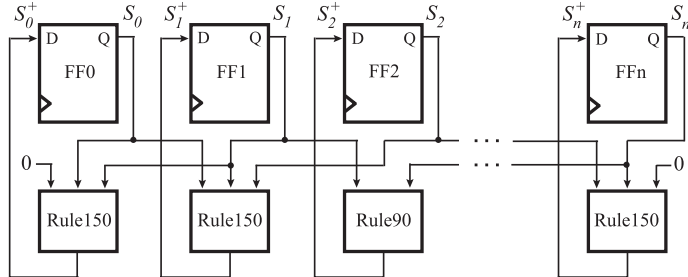

Fig. 9. CA-based random number generator

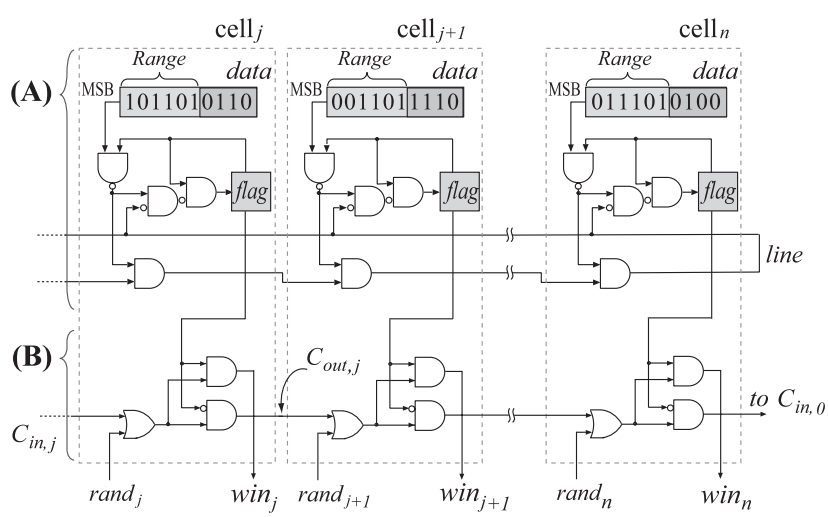

Fig. 10. RCM circuit; part (A) searches the SC, part (B) selects one individual from SC

by the rule sequence " $150-150-90-150 \cdots 90-150$ " produces a maximum-length cycle, i.e. it cycles through all $2^{16}$ possible bit patterns except the all 0 s pattern. It has also been shown that such a rule sequence has more randomness than a linear feedback shift register (LFSR) of corresponding length ${ }^{(21)}$. Figure 9 illustrates the random number generator with the above rule sequence.

5.1.2 Fitness Calculation Circuit The fitness calculation circuit computes the fitness values of the randomly generated population during initialization. Also, once a complete new population is generated by the mutation circuit, the fitness calculation circuit generates the fitness values for each of the generated individuals.

5.1.3 Selection Circuit Figure 10 illustrates the proposed selection circuit based on the RCM. This is a parallel processing circuit.

Part (A) searches the individuals of SC. First, shift left registers (data) store the fitness values of all the individuals, and flag of all cells are set to '1.' Secondly, when at least one of the MSBs is ' 1 ,' signal line becomes ' 0 .' Then, the data of MSB $=0$ is smaller than that of $\mathrm{MSB}=1$. Therefore, the flag of the data of MSB $=0$ changes to '0.' i.e. the data are no longer SC. Finally, data is shifted to the left. These operations are repeated Range times. The Range is the parameter which represents accuracy of the selection. In other words, the Range determines the threshold of the fitness. Part (A) takes a lot of SC when the Range is small.

Part (B) selects only one individual at random from the SC. Only one cell receives $\operatorname{rand}_{j}=1$, and others ' 0 ' at random by the random number generator. When the rand $_{j}=1$ and flag $_{j}=1$ or $C_{i n, j}=1$ and flag $_{j}=1$, $w_{i n}$ changes to ' 1 ,' otherwise ' 0 .' i.e. $j$-th individual is selected as the individual to be used in the next process

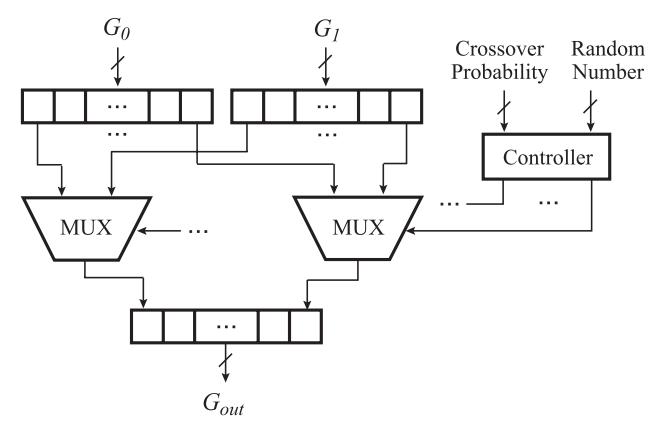

Fig. 11. Crossover circuit $^{(14)}$

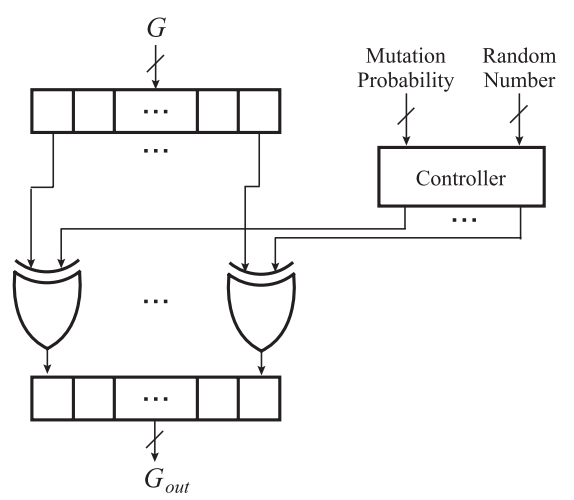

Fig. 12. Mutation circuit ${ }^{(14)}$

Table 3. The performance of GA hardware

\begin{tabular}{l|c}
\hline Operating frequency & $50 \mathrm{MHz}$ \\
\hline
\end{tabular}

\begin{tabular}{|l|l}
\hline Total gate of design & $200 \mathrm{~K}$ gates \\
\hline
\end{tabular}

of GAs. If the $\operatorname{flag}_{j}=0$ and rand $_{j}=1$, the chance to be selected is ceded to the next cell $\left(C_{\text {out }, j}=1\right)$.

5.1.4 Crossover Circuit ${ }^{(14)} \quad$ Figure 11 illustrates the crossover circuit which performs the singlepoint crossover operation. A crossover point depends on random values sent from the random number generator. When this circuit receives a selected pair of individuals from the selection circuit, it decides whether to perform crossover based on the probability of crossover which sent from the random number generator. When crossover is done, the new individual is sent to the mutation circuit.

5.1.5 Mutation Circuit ${ }^{(14)}$ Figure 12 illustrates the mutation circuit. When this circuit receives the individual from the crossover circuit, it decides whether to perform mutation based on the probability of mutation which sent from the random number generator in each bit. When mutation is done, the new individual is sent to the fitness calculation circuit for evaluation.

5.2 Hardware Simulations The problem in function maximization was applied to investigate the effects of the GA hardware embedded the RCM-based selection algorithm. In this paper, we used the following equation (7) as the evaluation function for simplicity (the sketch is shown in Fig. 13);

$$
f(x)=\left\{\begin{array}{cc}
2 x & (0 \leq x \leq 0.5) \\
-2 x+2 & (0.5<x \leq 1)
\end{array} .\right.
$$




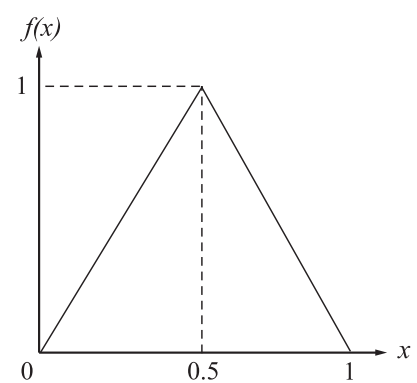

Fig. 13. The evaluation function of GA hardware

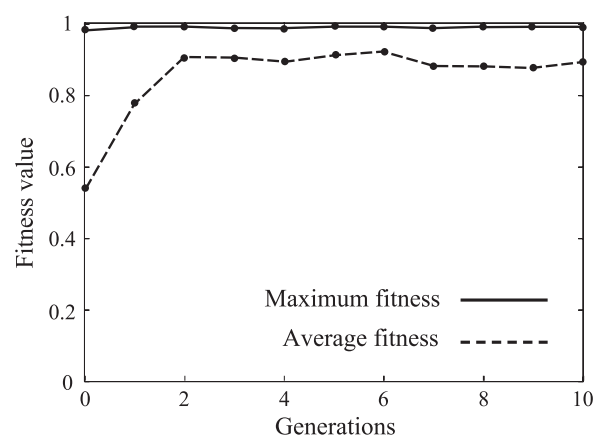

Fig. 14. The result of hardware simulation; these are average of 5 trials, where 10 generations are performed for 1 trial

We designed the GA hardware described in VHDL, and synthesized using a Mentor Graphics Precision Synthesis 2006 for a Xilinx XC2V6000-5 FPGA. A Xilinx ISE Foundation 8.1i was used for place and route. The hardware simulation using a Mentor Graphics ModelSim SE 6.2 is done. Table 3 shows the performance of the GA hardware embedded the RCM-based selection circuit.

Figure 14 illustrates the result of simulation. This result is represented as the maximum and average fitness values of each generation, and is average of 5 trials, where 10 generations are performed for 1 trial. As shown in Fig. 14, the average of fitness value gradually increased at each alternation of generations. Therefore, the GA hardware embedded the RCM-based selection circuit was considerable validity.

\subsection{Performance Comparisons}

5.3.1 Execution Time Figure 15 shows the comparison of the execution time for selecting one individual between the proposed RCM circuit and the previous works; the ordinary roulette wheel selection circuit by serial processing (Fig. 2(a), RW-s), the ordinary roulette wheel selection circuit by parallel processing (Fig. 2(b), RW-p), a roulette selection with binary search (BS) ${ }^{(13)(14)}$ and a simplified tournament selection $(\mathrm{STS})^{(15)}$, where the precision of fitness value is $16 \mathrm{bits}$.

Firstly, the RW-p and BS are parallel processing circuit, and are implemented as the combinational logic circuits. The STS is implemented by only two comparators. Thus, these circuits need no clocks.

Secondly, the execution time of the RCM is much shorter than the RW-s. As mentioned in Sec. 2.3, the RW $-\mathrm{S}$ is added the fitness values one by one until go-

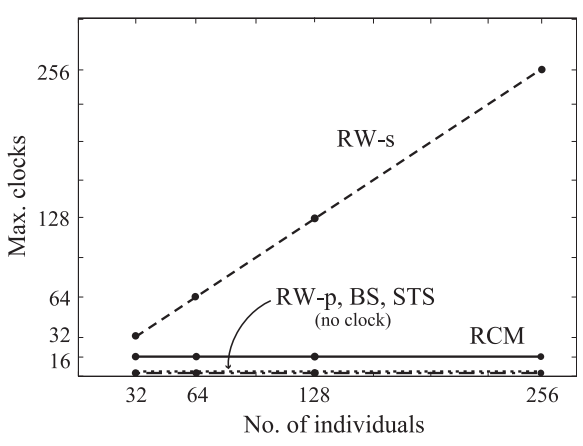

Fig. 15. Comparison of the execution time for selecting one individual by increase of individuals, where the fitness value is encoded in 16 bits

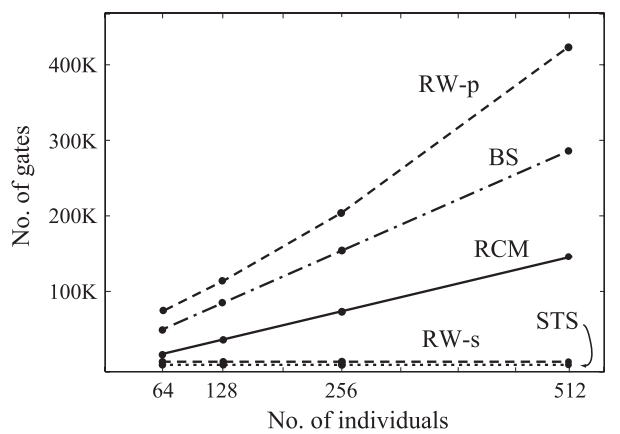

Fig. 16. Comparison of the circuit size of the proposed circuit and previous works

ing over the threshold. Therefore, the number of clocks which equal the number of individuals is needed until the individual is selected. Moreover, the execution time increases whenever the number of individuals increases. In contrast, the RCM selects the SC after the Range clocks are repeated, and then selects the individual from the $\mathrm{SC}$ by the combinational logic circuit. Therefore, the execution time is Range clocks, and is not more than the precision of fitness value (in this case, 16 clocks). In addition, the execution time is constant regardless of increase of the number of individuals because the RCM circuit is parallel processing circuit.

5.3.2 Circuit Size Figure 16 shows the comparison of the circuit size between the RCM and the previous works; RW-s, RW-p, BS and STS, where the individual and the fitness value are encoded in 64 bits and 16 bits, respectively.

Firstly, the circuit size of the RCM is much smaller than the RW-p and BS. The RW-p includes as many adders and comparators as the number of individuals (Fig. 2(b)) because this circuit has a parallel structure to expand the loop structure of Fig. 2(a). The BS is also a parallel processing circuit to narrow the search space of the target individuals by binary search. Therefore, the circuit size becomes much larger whenever the number of individuals increases. In contrast, the size of the $\mathrm{RCM}$ increases in proportion to the number of individuals as well as the RW-p and BS, yet the increase rate of the circuit size is few because the circuit size for one individual is small. 
Table 4. Comparison of the operating frequency

\begin{tabular}{c|c|c|c|c|c}
\hline & RWs & RWp & BS & STS & RCM \\
\hline Operating frequency $(\mathrm{MHz})$ & 108 & 25 & 54 & 133 & 51 \\
\hline
\end{tabular}

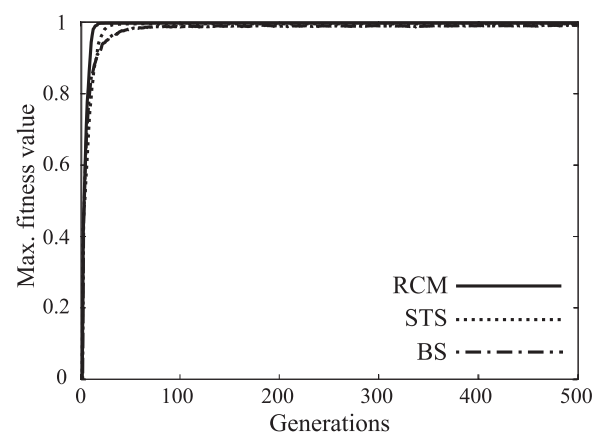

(a)

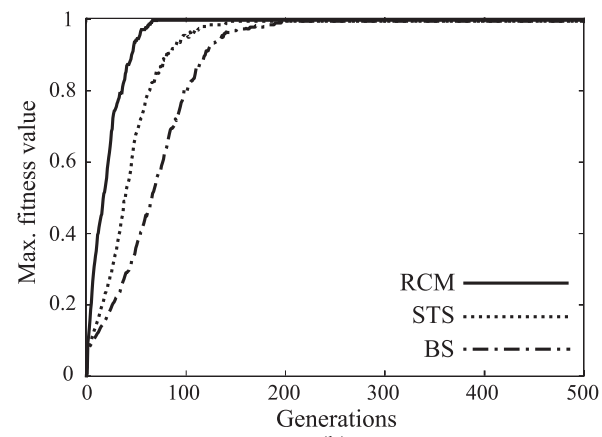

(b)

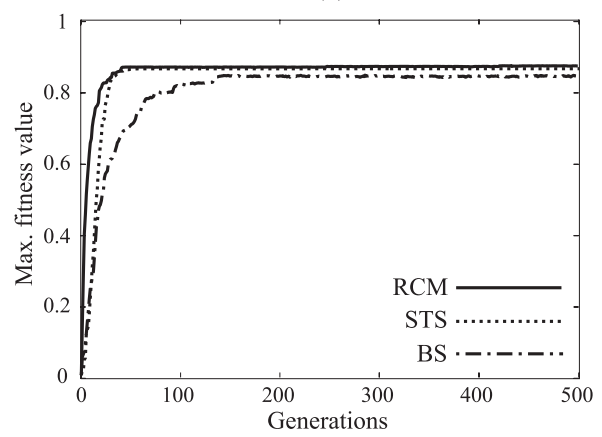

(c)

Fig. 17. Comparison of the selection schemes in the SGA; (a) parabola, (b) step function, (c) Shekel's foxholes; these are average of 100 trials, where 500 generations are performed for 1 trial

Secondly, the circuit size of the RW-s is considerably smaller than that of the RCM because the RW-s is implemented by an adder, an accumulator and a comparator (Fig. 2(a)). As with the RW-s, the circuit size of the STS is small because the STS is implemented by only two comparators. In addition, the circuit size is constant regardless of increase of the number of individuals because of the serial processing circuit.

5.3.3 Operating Frequency Table 4 shows the comparison of an operating frequency between the RCM and the previous works; RW-s, RW-p, BS and STS, where the fitness value is encoded in 16 bits. As shown in Table 4, the RCM circuit is not always high speed than other circuits. The low operating frequency is due to a chain circuit structure; the line signal chain and the $C_{i n}$ signal chain as shown in Fig. 10.

In order to reduce the delay time of the chain circuit,

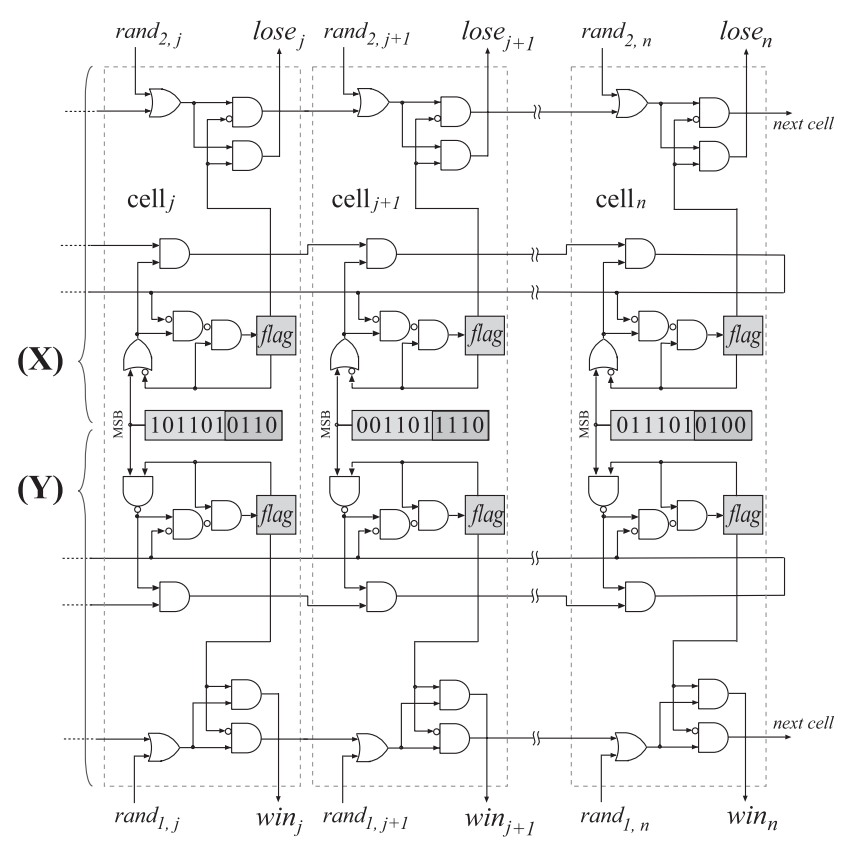

Fig. 18. RCM circuit for the SSGA; part (X) selects one individual with smaller fitness value, part (Y) selects one individual with larger fitness value

the RCM circuit is divided into some parts. For example, when the RCM circuit is divided into two parts, the delay time of each chain circuit are reduced to half because each length of the chain circuit is reduced by half. Thus, the operating frequency of the RCM circuit doubles.

5.3.4 Convergence Property The two type simulations were performed to compare the convergence properties between the RCM and the previous works (BS, STS); the simple GA (SGA) and a steady-state GA (SSGA).

SGA Simulation The BS and STS are embedded to the SSGA in original paper. In this section, we evaluated the convergence property by embedding BS and STS to the SGA. In the SGA, all individuals are updated in each generation. Hence these selection schemes are repeated until creating the same number of offspring as the individuals in population. The parameters of SGA were configured as shown in Table 1. And the SGA is applied to three De jong's test problems (Fig. 4).

Figure 17 illustrates the results of simulations. These results are represented as the maximum fitness values of each generation, and are average of 100 trials, where 500 generations are performed for 1 trial. As shown in Fig. 17 , we confirmed that the convergence property of the RCM is better than the other selection schemes.

SSGA Simulation The SSGA ${ }^{(22)}$ is an alternative to the SGA that replaces only a few individuals at a time, rather than an entire generation. In practice, the number of new strings created each generation is usually one or two. The new string(s) replace the string(s) selected from the population according to its fitness value. In this way the SSGA allows both parents and their offspring to coexist in same population.

When the RCM is embedded to SSGA, the RCM 


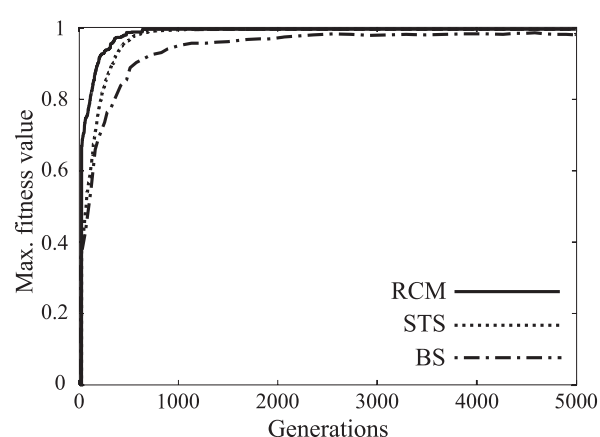

(a)

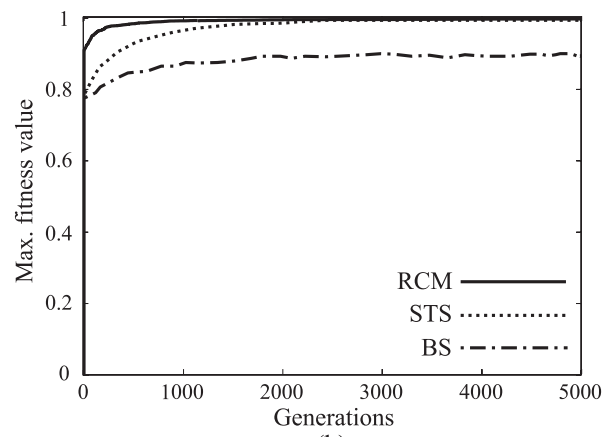

(b)

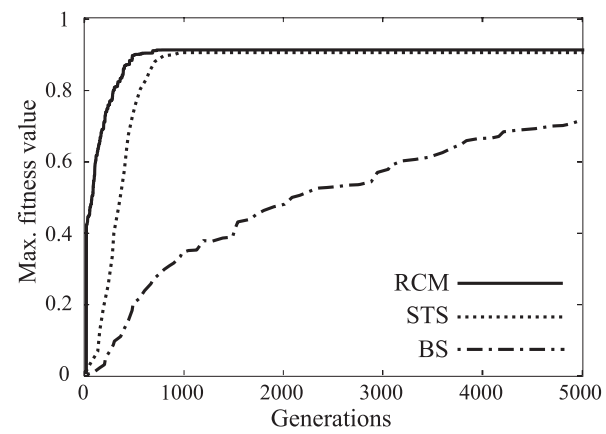

(c)

Fig. 19. Comparison of the selection schemes in the SSGA; (a) parabola, (b) step function, (c) Shekel's foxholes; these are average of 100 trials, where 5000 generations are performed for 1 trial

scheme is customized as follows for the SSGA;

(1) Two individuals with larger fitness values are selected $\left(G_{L 1}, G_{L 2}\right)$.

(2) Two individuals with smaller fitness values are selected $\left(G_{S 1}, G_{S 2}\right)$.

(3) The genetic operations are applied to $G_{L 1}$ and $G_{L 2}\left(G_{L 1}^{\prime}, G_{L 2}^{\prime}\right)$.

(4) $G_{S 1}$ and $G_{S 2}$ are replaced with $G_{L 1}^{\prime}$ and $G_{L 2}^{\prime}$. The RCM circuit customized as shown in Fig. 18 is able to select the individual with smaller fitness value as well as larger fitness value although the circuit size increases somewhat.

The parameters of SSGA were configured as shown in Table 1. And the SSGA is applied to three De jong's test problems (Fig. 4).

Figure 19 illustrates the results of simulations. In Fig. 19 , these results are represented as the maximum fitness values of each generation, and are average of 100 trials, where 5000 generations are performed for 1 trial. As shown in Fig. 19, we confirmed that the convergence property of the RCM is better than the other selection schemes.

The BS scheme narrowed the search space of the target individuals in roulette wheel selection by binary search to reduce of execution time. Therefore the BS scheme does not always select the individual with larger fitness value. The STS scheme selects two individuals at random, and the better one reproduces to next generation. Thus, as with the BS, the STS scheme does not always select the individual with larger fitness value. In contrast, the RCM scheme certainly selects the individual with larger fitness value. Therefore we considered that the convergence property of the RCM is better than the other selection schemes in both SGA and SSGA. However, it should be considered that the circuit size of the $\mathrm{RCM}$ somewhat increases.

\section{Discussions}

6.1 Variable Range A difference between individuals does not appear within the Range bits because there is not so much of the difference of the fitness value between individuals in the final phase of the convergence. In these circumstances, the proposed method operates as a random search. If a suitable Range bits for problem can be set in advance, it is possible to search for an optimal solution by the random search.

On the other hand, if the suitable Range bits for the problem cannot be set in advance, the other way is that a length of the Range is increased in the final phase of the convergence. As a result, the proposed method does not operate as a random search in the final phase, and can search the optimal solution.

6.2 Combinatorial Optimization Three test functions presented in Fig. 4(a)(b)(c) have been firstly proposed by De Jong ${ }^{(17)}$ to measure the performance of GA. The test environment includes functions which are convex (parabola), discontinuous (step function) and multimodal (Shekel's foxholes).

Because the step function has a discrete-valued data, the optimal solution is obtained by combining the discrete-valued data. Hence, the step function is a combinatorial optimization problem.

In contrast, the Shekel's foxholes has a continuousvalued data. However, this function has many local optima, and seems the discrete function. Therefore, the Shekel's foxholes as well as the step function is considered the combinatorial optimization problem. As we mentioned before, the RCM-based selection method has effectiveness of these test functions. As a result, the RCM-based selection method has effectiveness for not only the functional optimization problem but also the combinatorial optimization problem. i.e. a traveling salesman problem and a knapsack problem.

\section{Conclusions}

In this paper, we proposed the new selection circuit based on the rough comparison method, and evaluated effects of the proposed circuit in terms of the execution time and circuit size. The software simulations results showed that the proposed selection scheme has a perfor- 
mance better than the ordinary roulette wheel selection scheme. The hardware implementation result showed that the proposed circuit has a considerable validity. In considering that the circuit size of the RCM increases somewhat, the convergence property of the proposed selection scheme is better than the other previous works. From what has been discussed above, we can conclude that the proposed circuit suits well for the GA hardware.

\section{Acknowledgment}

This work was partially supported by a 21st Century Center of Excellence Program, "World of Brain Computing Interwoven out of Animals and Robots (PI: T. Yamakawa)" granted in 2003 to Department of Brain Science and Engineering, Graduate School of Life Science and Systems Engineering, Kyushu Institute of Technology by Japan Ministry of Education, Culture, Sports, Science and Technology.

(Manuscript received June 11, 2007, revised Oct. 26, 2007)

\section{References}

(1) J.H. Holland: "Adaptation in Natural and Artificial Systems", University of Michigan Press (1975)

( 2 ) D.E. Goldberg: "Genetic Algorithms in Search, Optimization \& Machine Learning", Addison-Wesley (1989)

( 3 ) D.E. Goldberg, J. Borgerson, A. Vaughn, K. Hawley, C. Cunningham, J. Milner, K. Zacarias, B. Wagus, R. Gadient, B. Sutton, M. Pelikan, F. Rothlauf, and E. Cantú-Paz: "Genetic Algorithms: A Bibliography", IlliGAL Report, No.2000037 (2000)

(4) A. Maruyama, N. Shibata, Y. Murata, K. Yasumoto, and M. Ito: "P-Tour: A Personal Navigation System with Travel Schedule Planning and Route Guidance Based on Schedule", IPSJ Journal, Vol.45, No.12, pp.2678-2687 (2004) (in Japanese)

( 5 ) H. Sakanashi, M. Iwata, and T. Higuchi: "Lossless Compression of Very High Resolution Bi-level Images Using Genetic Algorithm", IPSJ Journal, Vol.45, No.5, pp.1460-1470 (2004) (in Japanese)

(6) B.C.H. Turton, T. Arslan, and D.H. Horrocks: "A Hardware Architecture for Parallel Genetic Algorithms for Image Registration", Proc. of IEE Colloq. on Genetic Algorithms in Image Processing and Vision, pp.11/1-11/6 (1994)

( 7 ) B.C.H. Turton and T. Arslan: "A Parallel Genetic VLSI Architecture for Combinatorial Real-Time Applications - Disk Scheduling", Conf. Proc. on Genetic Algorithms in Engineering Systems: Innovations and Applications, pp.493-500 (1995)

(8) P. Graham and B. Nelson: "Genetic Algorithms in Software and in Hardware - A Performance Analysis of Workstations and Custom Computing Machine Implementations", Proc. of IEEE Symp. on FPGAs for Custom Computing Machines, pp.216-225 (1996)

( 9 ) Y. Yano, T. Hashiyama, and S. Okuma: "On-line Filter Generation for Binary Image Processing Using FPGA", Proc. 1999 IEEE Int'l Conf. on Systems, Man and Cybernetics, Vol.5, pp.565-570 (1999)

(10) S.D. Scott, A. Samal, and S. Seth: "HGA: A Hardware-Based Genetic Algorithm", Proc. of 1995 ACM/SIGDA 3rd Int'l Symp. on FPGA, pp.53-59 (1995)

(11) I.M. Bland and G.M. Megson: "Implementing a Generic Systolic Array for Genetic Algorithms", Proc. 1st Online Workshop on Soft Computing, pp.268-273 (1996)

(12) B. Shackleford, G. Snider, R.J. Carter, E. Okushi, M. Yasuda, K. Seo, and H. Yasuura: "A High-Performance, Pipelined, FPGA-Based Genetic Algorithm Machine", Journal of Genetic Programming and Evolvable Machines, Vol.2, No.1, pp.33-60 (2001)
(13) H. Asada, H. Sugiura, T. Kawai, H. Ando, and T. Shimada: " $H^{3}$ : High-speed Hardware for Human-like genetic algorithm", Proc. of 3rd Int'l Symp. on Artificial Life, and Robotics, pp.190-195 (1998)

(14) O. Kitaura, H. Asada, M. Matsuzaki, T. Kawai, H. Ando, and T. Shimada: "A Custom Computing Machine for Genetic Algorithms without Pipeline Stalls", Trans. of SICE, Vol.35, No.11, pp.1496-1504 (1999) (in Japanese)

(15) N. Yoshida, T. Yasuoka, T. Moriki, and T. Shimokawa: "VLSI Hardware Design for Genetic Algorithms and Its Parallel and Distributed Extensions", Int'l Journal of KnowledgeBased Intelligent Engineering Systems, Vol.5, No.1, pp.14-21 (2001)

(16) H. Tamukoh, K. Horio, and T. Yamakawa: "Fast Learning Algorithms for Self-Organizing Map Employing Rough Comparison WTA and its Digital Hardware Implementation", IEICE Trans. on Electronics, Vol.E87-C, No.11, pp.1787-1794 (2004)

(17) K.A. De Jong: An Analysis of the Behavior of a Class of Genetic Adaptive Systems, Ph.D. dissertation, Univ. of Michigan (1975)

(18) A. Törn and A. Zilinskas: "Global Optimization", Lecture Notes in Computer Science, No.350, Springer-Verlag, Berlin (1989)

(19) D. Whitley: "Fundamental Principles of Deception in Genetic Search", Foundations of Genetic Algorithms, ed. G. Rawlins, Morgan Kaufmann (1991)

(20) M. Serra, T. Slater, J.C. Muzi, and D.M. Miller: "The Analysis of One-Dimensional Linear Cellular Automata and Their Aliasing Properties", IEEE Trans. on Computer-Aided Design of Integrated Circuits and Systems, Vol.9, No.7, pp.767778 (1990)

(21) P.D. Hortensius, R.D. McLeod, and H.C. Card: "Parallel Random Number Generation for VLSI Systems Using Cellular Automata", IEEE Trans. on Computers, Vol.38, No.10, pp.1466-1473 (1989)

(22) G. Syswerda: "A study of reproduction in generational and steady-state genetic algorithms", G.J.E. Rawlins, ed. Foundations of Genetic Algorithms, Morgan Kaufmann, pp.94101 (1991)

Tomokazu Hiratsuka (Student Member) was born in 1979.

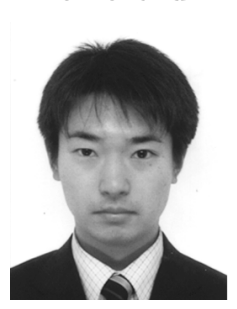
He received the B. Eng. degree and the M. Eng. degree from Kyushu Institute of Technology, Japan in 2002 and 2004, respectively. He is currently working towards the Ph.D. degree in brain science and engineering of Kyushu Institute of Technology, Japan. He is a student member of IEEJ and the Institute of Electronics, Information and Communication Engineers (IEICE).

Hakaru Tamukoh (Non-member) was born in $1978 . \mathrm{He}$

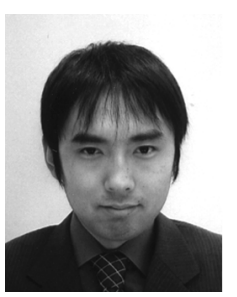
received the B. Eng. degree in 2001 from Miyazaki University, and the M. Eng. degree and the Ph.D. degree in 2003 and 2006, respectively from Kyushu Institute of Technology, both in Japan. He was as a research fellow of the Graduate School of Life Science and Systems Engineering, Kyushu Institute of Technology, Japan from 2006 to 2007. He is an assistant professor of the Institute of Symbiotic and Technology, Tokyo University of Agriculture and Technology, Japan since 2007. He is a member of the Institute of Electronics, Information and Communication Engineers (IEICE) and Japan Society for Fuzzy Theory and Intelligent Informatics (SOFT). 
Keiichi Horio (Non-member) was born in 1972. He received the B. Eng. degree, M. Eng. degree and the Ph.D. degree from Kyushu Institute of Technology, Japan in 1996, 1998 and 2001, respectively. He was a research fellow of Japan Society for Promotion of Science at Kyushu Institute of Technology from 2001 to 2003 . He was an assistant professor of the Graduate School of Life Science and Systems Engineering, Kyushu Institute of Technology, Japan from 2003 to 2007. He is an associate professor of the Graduate School of Life Science and Systems Engineering, Kyushu Institute of Technology, Japan since 2007. He is a member of the Institute of Electronics, Information and Communication Engineers (IEICE) and Japan Society for Fuzzy Theory and Intelligent Informatics (SOFT).
Takeshi Yamakawa (Non-member) was born in 1946. He

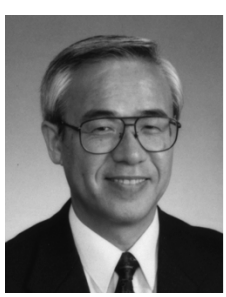
received the B. Eng. degree in electronics engineering in 1969 from Kyushu Institute of Technology, Tobata and M. Eng. degree in electronics engineering in 1971 from Tohoku University, both in Japan. He received the Ph.D. degree for his studies on electrochemical devices in 1974 from Tohoku University, Japan. He was an Associate Professor at Kumamoto University. He joined the faculty of Computer Science and Systems Engineering, Kyushu Institute of Technology (KIT), Iizuka, Japan in April 1989 as a full professor. He is a professor of the Graduate School of Life Science and Systems Engineering, Kyushu Institute of Technology since 2001. He is also the chairman of Fuzzy Logic Systems Institute (FLSI), the foundation established by himself in 1990. His main research interest lies on hardware implementation of fuzzy systems, fuzzy neural networks, and chaotic systems, and also on the micromachining and the micro-electrophoresis device. He holds 11 patents in U.S.A., 4 patents in Europe, 1 patent in Australia and 1 patent in Taiwan, and he has also applied for more than 80 patents in Japan. He received the International MOISIL Prize and the Gold Medal in the field of Fuzzy Systems Engineering in 1994, Distinguished Service Prize from Biomedical Fuzzy Systems Association in 1998, Doctorate Honoris Causa from University of Pitesti, Romania in 1999, Lifetime Achievement Award from WAC in 2000, Gabor Award from IJCNN 2001, Fellowship Award from Japan Society for Fuzzy Theory and Intelligent Informatics in 2003. He is a member of IFSA and other 9 academic institutes. He is acting as a member of editorial board and a regional editor of 19 international professional journals. He contributed more than 50 international conferences as an organizer or a member of organizing/programming committee. 